\title{
Pharmacological evaluation of rat dorsal root ganglion neurons as an in vitro model for diabetic neuropathy
}

This article was published in the following Dove Press journal: Journal of Pain Research

II February 2011

Number of times this article has been viewed

\author{
Eve Peeraer ${ }^{1,2}$ \\ An Van Lutsenborg ${ }^{3}$ \\ An Verheyen ${ }^{1,4}$ \\ Raf De Jongh ${ }^{5}$ \\ Rony Nuydens' \\ Theo F Meert ${ }^{1,2}$ \\ Johnson \& Johnson Pharmaceutical \\ Research and Development, Beerse, \\ Belgium; ${ }^{2}$ University Hasselt, Hasselt, \\ Belgium; ${ }^{3}$ Free University Brussels, \\ Brussels, Belgium; ${ }^{4}$ The Vesalius \\ Research Center, University of \\ Leuven, Leuven, Belgium; ${ }^{5}$ Intensive \\ Care and Emergency Care, Ziekenhuis \\ Oost-Limburg, Genk, Belgium
}

Correspondence: Eve Peeraer Johnson \& Johnson Pharmaceutical Research and Development, Neuroscience Division, Janssen Pharmaceutica NV,

Turnhoutseweg 30,

2340 Beerse, Belgium

Tel +32 I460 3844

Fax $+32 \quad 14603753$

Email epeerael@its.jnj.com
Background: Diabetic neuropathy is a complication of diabetes mellitus that develops in about $50 \%$ of people with diabetes. Despite its widespread occurrence and devastating effects, this complication is still not fully understood, and there is no treatment available to prevent its development.

Methods: In this study, immunocytochemistry for activating transcription factor 3, a marker for cell injury, was used to investigate the stress response in dorsal root ganglion neurons in both in vitro and ex vivo models of diabetic neuropathy.

Results: Our findings showed increased activating transcription factor 3 expression in hyperglycemic culture conditions and in dorsal root ganglion neurons isolated from diabetic rats. Glial cell line-derived neurotrophic factor, a substance with known neuroprotective properties, was able to reduce diabetes mellitus-induced neuronal stress in vitro, while gabapentin and carbamazepine, currently used to treat neuropathic pain, showed only limited effects.

Conclusion: Growth factors may have a therapeutic benefit as neurotrophic agents in the treatment of diabetic peripheral neuropathy, but gabapentin and carbamazepine have no direct protective effect on sensory neurons. This research also indicates that immunocytochemistry for activating transcription factor 3 is a valuable tool for evaluation of pharmacological substances in dorsal root ganglion cultures.

Keywords: diabetic peripheral neuropathy, dorsal root ganglion, activating transcription factor 3, glial cell line-derived neurotrophic factor, anticonvulsants

\section{Introduction}

Diabetic peripheral neuropathy is a common chronic complication of diabetes mellitus that develops in about $50 \%$ of patients affected with type 1 or 2 diabetes. ${ }^{1}$ This neuropathy is associated with a spectrum of structural changes in the peripheral nervous system, including axonal degeneration, paranodal demyelination, and loss of myelinated fibers. ${ }^{2,3}$ Although the exact pathogenesis of diabetic peripheral neuropathy is unknown, results of the Diabetes Control and Complications Trial have indicated that hyperglycemia is a key player in the development of the disorder. ${ }^{4,5}$ In addition to hyperglycemia, neurotrophic factor deficiency has received increasing attention as being important in the pathology of diabetic peripheral neuropathy. There is evidence indicating that the loss of neurotrophic support seen in diabetes mellitus has detrimental consequences for the survival and regenerative responses of neurons. ${ }^{6,7}$

Despite the devastating effects and widespread occurrence of diabetic peripheral neuropathy, there are still no treatments available to prevent its development, other than the overall control of the diabetic condition itself. Therefore, it is necessary to study 
the pathological mechanisms underlying diabetic peripheral neuropathy to understand better the development of this complication and to look for more specific disease-modifying treatments.

In this study, adult rat dorsal root ganglion (DRG) neurons were used as an in vitro model to investigate diabetic neuropathy. The sensory perikaryons of the peripheral nervous system, which are the target tissues in the pathophysiology of diabetic peripheral neuropathy, are located within the DRG. When cultured rat DRG neurons are exposed to a variety of toxic insults, the neurons undergo morphological and biochemical changes which closely mimic toxin-mediated damage in adult animals and humans. ${ }^{8,9}$ Therefore, these cultured DRG neurons provide a robust and relevant in vitro model to investigate diabetic neuropathy. Furthermore, DRG neurons can be extracted from normal animals and exposed in vitro to toxins, and DRG neurons from diabetic animals can be evaluated ex vivo. As such, this approach allows us to link in vitro analysis to the in vivo events that occur during the development of peripheral neuropathy associated with diabetes.

The goal of this research was to explore further the role of glial cell line-derived neurotrophic factor (GDNF) in diabetic peripheral neuropathy and to compare the effects of this growth factor with those of gabapentin and carbamazepine, both anticonvulsants currently in use to treat neuropathic pain. ${ }^{10,11}$ GDNF was originally purified as a potent neurotrophic factor that enhances the survival of midbrain dopaminergic neurons. ${ }^{12}$ Subsequent studies showed that GDNF also supports the survival of other types of neurons, including both motor and sensory neurons. ${ }^{13,14}$ The potent neurotrophic effects of GDNF, together with the reduced expression and transport of growth factors seen in animal models of diabetes, suggest a promising role for GDNF in the treatment of diabetic peripheral neuropathy, and this was also investigated in this study.

Gabapentin and carbamazepine are only symptomatic treatments available for diabetic neuropathy, and are not believed to inhibit or reverse the pathological processes involved. Both drugs inhibit the excessive neuronal activity seen in neuropathic pain. Carbamazepine decreases abnormal electrical impulses in the nerve by blocking sodium channels, ${ }^{15}$ whereas gabapentin is believed to interact with the alpha-2 subunit of the calcium channel to inhibit glutamate release. ${ }^{11,16}$

To evaluate the potential therapeutic effects of GDNF, gabapentin, and carbamazepine, the expression of activating transcription factor 3 (ATF3) was measured in both in vitro and ex vivo models of diabetic neuropathy. ATF3 is a member of the activating transcription factor/cAMP response element-binding family of transcription factors and is induced by a variety of stress signals in both neuronal and nonneuronal cells. ${ }^{17-20}$ ATF3 is an immediate early gene, and a change in its expression level is among the earliest events reported after injury, suggesting a potential role in the stress response. This protein can either activate or repress transcription, depending on whether it forms homodimers or heterodimers with Jun proteins. ${ }^{17,21}$ Because several studies have demonstrated that ATF3 is induced in sensory and motor neurons after peripheral nerve injury, this transcription factor has been generally accepted as a marker for neuronal stress. ${ }^{20,22-26}$ However, all previous studies have quantified ATF3 expression immunohistologically, and none have used this marker to assess neuronal stress in cultured cells. The advantage of using cultured DRG neurons for ATF3 quantification instead of using whole DRGs is the ability to add different compounds to the cultured neurons, and in this way search for pharmacological substances able to reduce the stress associated with diabetes mellitus. Therefore, in this study we investigated whether immunocytochemistry for ATF3 is an effective tool for evaluation of the effects of pharmacological substances in DRG cultures.

\section{Experimental procedures Rat models}

Male Sprague-Dawley rats (Harlan Laboratories, Horst, The Netherlands) weighing 240-260 g were fed a standard rodent diet and had access to water ad libitum. Diabetes was induced by a single intraperitoneal injection of streptozotocin (SigmaAldrich, St. Louis, MO) $65 \mathrm{mg} / \mathrm{kg}$ after an overnight fast (starvation for 20-24 hours). Blood samples for measurement of glucose were taken from the tail vein 48 hours after streptozotocin injection. Rats with a blood glucose level above $500 \mathrm{mg} / \mathrm{dL}$ were considered diabetic and used for further experiments. Control animals received buffer solution instead. All studies were performed in accordance with institutional ethical guidelines for the care and use of laboratory animals, and the protocols were approved by the Local Animal Care Ethics committee.

\section{Paw pressure test}

Mechanical hyperalgesia was quantified using an AnalgesyMeter (Ugo-Basile, Comerio, Italy). Increasing pressure was applied using a cone-shaped plunger on the dorsal area of 
the hind paw. The nociceptive threshold was defined as the force at which the rat attempted to withdraw its paw, vocalized, or struggled. The cutoff force was set at $250 \mathrm{~g}$.

\section{Isolation of DRG neurons}

The rats were sacrificed by carbon dioxide inhalation. Immediately thereafter, DRG neurons were dissected from the spinal column and collected in phosphate-buffered saline containing $1 \mathrm{~g} / \mathrm{L}$ glucose. After isolation, the ganglia were enzymatically dissociated by incubation at $37^{\circ} \mathrm{C}$ for 45 minutes in medium containing $0.5 \%$ collagenase (Roche Diagnostics, Mannheim, Germany) followed by $0.25 \%$ trypsin (Gibco, Paisley, UK) in phosphate-buffered saline for 30 minutes. The ganglia were then collected in Dulbecco's modified Eagle's medium (Gibco) enriched with fetal calf serum, nonessential amino acids, sodium bicarbonate, and L-glutamine. The ganglia were mechanically dissociated into single cells by titration through flame-constricted Pasteur pipettes of decreasing tip diameter. To purify the cultures, the cell suspension was placed in a Petri dish previously coated with fetal calf serum (HyClone, Carlsbad, CA) for 90 minutes at $37^{\circ} \mathrm{C}$. The DRG neurons were then collected and plated into $10 \mu \mathrm{g} / \mathrm{mL}$ poly-L-lysine-coated 96-well plates.

\section{DRG cultures and treatment}

DRG neurons were cultured in Neurobasal medium (Gibco) supplemented with B27 supplement (Gibco). This commercially available medium contains $30 \mathrm{mM}$ glucose. DRG neurons were cultured for at least 24 hours in medium containing the indicated compounds. A GDNF concentration in the $0.1-10.0 \mathrm{ng} / \mathrm{mL}$ range was used because previous studies had shown these doses to be effective. ${ }^{27,28}$ Lower doses were not effective, and higher doses showed aberrant stimulation (data not shown).

Clinical trials have shown that plasma concentrations of $2-11 \mu \mathrm{g} / \mathrm{mL}$ (corresponding to $23-64 \mu \mathrm{M}$ ) for gabapentin and 4-16 $\mu \mathrm{g} / \mathrm{mL}$ (corresponding with 17-68 $\mu \mathrm{M}$ ) for carbamazepine are required to produce a therapeutic effect. ${ }^{16,29}$ In this study, DRG neurons were treated for 24 hours with concentrations of gabapentin and carbamazepine in this therapeutic range $(10 \mu \mathrm{M}$ and $100 \mu \mathrm{M}$, respectively). To ensure that gabapentin and carbamazepine had no effect on ATF3 expression, and because an in vitro model was used, higher concentrations of these anticonvulsant drugs were also tested $(200 \mu \mathrm{M}$ and $250 \mu \mathrm{M}$, respectively).

\section{Immunocytochemistry}

DRG neurons were fixed for 10 minutes using $0.5 \%$ Triton X100 (Sigma) and 0.5\% glutaraldehyde (Fluka Chemie, Buchs, Switzerland) in PHEM buffer (60 mM PIPES, $10 \mathrm{mM}$ EGTA, and $2 \mathrm{mM} \mathrm{MgCl}_{2}, \mathrm{pH}$ 6.0). The cells were washed in PHEM before addition of $0.5 \%$ Triton X100 alone for 30 minutes. The cells were again washed with PHEM buffer, and then $1 \mathrm{mg} / \mathrm{mL} \mathrm{NaBH}_{4}$ (Sigma-Aldrich Chemie, Munich, Germany) dissolved in PHEM buffer was added for 10 minutes. This was followed by a wash in PHEM buffer. Prior to addition of primary antibodies, the cells were incubated for 30 minutes in phosphate-buffered saline/bovine serum albumin $0.1 \%$ (Sigma) buffer containing 5\% normal goat serum (Sigma). The cells were dual-labeled using a polyclonal anti-ATF3 antibody (1:800 dilution in phosphatebuffered saline/bovine serum albumin; Santa Cruz Biotechnology, Santa Cruz, CA) as a neuronal stress marker and the monoclonal antineurofilament antibody, SMI32 (1:1000 dilution in phosphate-buffered saline/bovine serum albumin; Sternberger Monoclonals Inc, Baltimore, MD) as a neuronal marker. Following overnight incubation at room temperature, the cells were washed and incubated with the secondary antibodies, Alexa Fluor 555 goat antirabbit IgG (Invitrogen, Carlsbad, CA) and Alexa Fluor 488 goat antimouse IgG (Invitrogen). Then the cells were washed with phosphatebuffered saline/bovine serum albumin, and DAPI (4',6diamidino-2-phenylindole; Sigma) was added for five minutes as a nuclear counterstain. After washing, the percentage of ATF3-positive neurons was determined in each specific culture condition using fluorescence microscopy. Only SMI32positive cells were considered, and irrespective of their size.

\section{Quantitative RT-PCR}

The RNeasy kit (Qiagen, Hilden, Germany) was used to extract RNA from whole DRGs at the L4, L5, and L6 levels. Approximately $1 \mu \mathrm{g}$ of RNA was transcribed to cDNA using the QuantiTect reverse transcription kit (Qiagen). Gene expression was analyzed by the 7500 Fast real-time polymerase chain reaction (RT-PCR) system (Applied Biosystems, Foster City, CA) and normalized to $ß$-actin expression levels.

\section{Experiments performed}

\section{Determination of optimal glucose levels} in control cultures

DRGs (from all levels, ie, cervical, thoracic, and lumbar) were isolated from control rats and cultured for 24,48 , or 72 hours in medium containing either $10 \mathrm{mM}$ or $30 \mathrm{mM}$ 
glucose. The percentage of ATF3-positive neurons was determined for each condition $(n=6$ wells $/ 3$ rats, with at least 100 neurons quantified in every well).

\section{ATF3 induction in DRG cultures exposed to} hyperglycemic conditions

DRG neurons (from all spinal levels) isolated from control rats were exposed to different glucose levels for 24 hours and labeled for ATF3. The percentage of ATF3-positive neurons was determined for each condition $(n=6$ wells $/ 3$ rats).

\section{Time course of ATF3 expression in DRG cultures} from diabetic rats

Rats were treated with streptozocin, and DRG neurons from all spinal levels were isolated and placed in identical culture conditions at the indicated time points. After 24 hours, the cells were fixed and processed for ATF3 quantification ( $\mathrm{n}=18$ wells from 3 rats/time point).

\section{RT-PCR for ATF3, c-jun, and c-fos in lumbar} DRGs from diabetic rats

Whole DRGs from levels L4, L5, and L6 were dissected from diabetic and control rats and immediately frozen. RNA extraction, cDNA preparation, and quantitative RTPCR analysis for ATF3, c-jun, and c-fos were conducted ( $\mathrm{n}=6$ rats).

\section{Effect of GDNF, gabapentin, and carbamazepine} on acute hyperglycemia-induced ATF3 expression in DRG cultures

DRGs from all levels were isolated from control animals. After one day in culture, the cells were treated for four hours with the compounds at the indicated concentrations. The concentration of glucose in the medium was increased to $60 \mathrm{mM}$, and 24 hours later the cultures were processed for ATF3 quantification ( $n=6$ wells $/ 3$ rats). The percentage of positive neurons was determined and the relative increase calculated for each condition when compared with normal culture conditions.

\section{Effect of GDNF, gabapentin, and carbamazepine} ex vivo on ATF3 expression in DRG cultures from diabetic rats

DRGs from all levels were isolated from diabetic animals 7-13 weeks after streptozocin injection. Immediately after isolation, the cells were treated with the compounds at the indicated concentrations, and 24 hours later, the cultures were processed for ATF3 quantification. The percentage of ATF3-positive neurons was determined, and the relative changes calculated for each condition when compared with compound-treated cultures from nondiabetic control rats ( $\mathrm{n}=6 \mathrm{wells} / 3$ rats).

\section{Effect of in vivo GDNF on ATF3 expression in DRG cultures from diabetic rats}

After 10 weeks of being in a diabetic state, the rats received a subplantar injection of GDNF $1 \mathrm{mg} / \mathrm{mL}$ or phosphatebuffered saline in the left paw for three consecutive days. L4, L5, and L6 DRG neurons from the left side were then isolated. After 24 hours in culture, the cells were fixed and processed for ATF3 quantification ( $n=6$ wells $/ 3$ rats).

\section{Effect of GDNF treatment on diabetes-induced pain hypersensitivity in vivo}

Two-week diabetic rats received a subplantar injection of GDNF $1 \mathrm{mg} / \mathrm{mL}$ or phosphate-buffered saline in the left paw for three consecutive days. The paw pressure test was used to investigate mechanical hyperalgesia. The animals were followed up from one day before until four weeks after streptozocin injection ( $\mathrm{n}=10 \mathrm{rats} /$ group).

\section{Statistical analysis}

The results are shown as the mean \pm standard error of the mean. To calculate the significant difference between two groups, a Student's $t$-test was used. To test whether the mean values were significantly different between more than two groups, we used a one-way analysis of variance test, followed by a post hoc Holm-Sidak multiple comparisons test. A $P$ value of $<0.05$ was set as the level of statistical significance.

\section{Results}

\section{Determination of the optimal glucose levels in control cultures}

We evaluated two commonly used concentrations of glucose in the culture medium to determine the concentration of glucose required for optimal growth of DRG neurons. Several studies of adult rat DRG cultures have used $10 \mathrm{mM}$ glucose in control conditions. ${ }^{30,31}$ Others state that optimal DRG survival and neurite growth require $25-30 \mathrm{mM}$ basal glucose $e^{9,32}$ because sensory neurons have high metabolic requirements. This higher concentration of glucose is often used in embryonic DRG cultures. To compare both conditions, DRG neurons were isolated from rats and cultured 
in Neurobasal medium containing either $10 \mathrm{mM}$ or $30 \mathrm{mM}$ glucose. After 24, 48, or 72 hours in culture, the neurons were fixed and used for ATF3 quantification (Figure 1). There was no significant difference in ATF3 expression in DRG neurons cultured in medium containing 10 or $30 \mathrm{mM}$ glucose for the three time points (Figure 2). However, we did see a slightly higher percentage of ATF3-positive neurons in medium containing $10 \mathrm{mM}$ of glucose after 48 and 72 hours in culture, indicating that $30 \mathrm{mM}$ glucose might be able to meet the high metabolic requirements of DRG neurons better after a longer culture period. Because most of our subsequent work required a culture period of 48 hours, we used medium containing $30 \mathrm{mM}$ glucose for all experiments.

\section{Induction of ATF3 in DRG cultures exposed to hyperglycemic conditions}

To evaluate the effect of high glucose concentrations, cultured DRG neurons were exposed for 24 hours to hyperglycemic culture conditions. The cells were harvested in culture medium containing a total glucose concentration of 30-110 mM. Exposure to the higher glucose concentrations induced an increase in the number of ATF3-positive cells when compared with control cultures ( $30 \mathrm{mM}$ glucose), with significant changes from $60 \mathrm{mM}$ glucose onwards (Figure 3 ). A concentration of $110 \mathrm{mM}$ glucose showed no significant increase in ATF3 expression. This underestimation of cellular stress is most likely due to cell loss.

\section{ATF3 expression in DRG cultures from diabetic rats}

To investigate whether there is a higher level of neuronal stress in diabetic conditions in vivo and to document the time course of this process, DRG neurons were isolated from rats at different time points after streptozotocin injection. ATF3 expression was

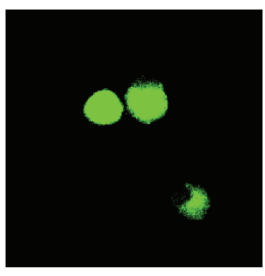

Neurofilament

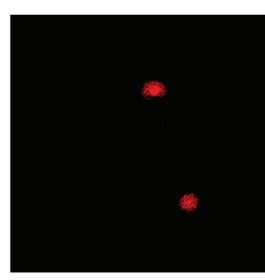

ATF3

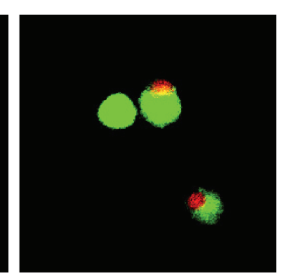

Composed image
Figure I Example of dorsal root ganglion neurons stained for ATF3 (red) and neurofilament (green). Dorsal root ganglion neurons were isolated from control rats and plated in 96-well plates. After one day in culture, the cells were incubated in $60 \mathrm{mM}$ glucose for 24 hours and stained for ATF3 and neurofilament.

Abbreviation: ATF3, activating transcription factor 3.

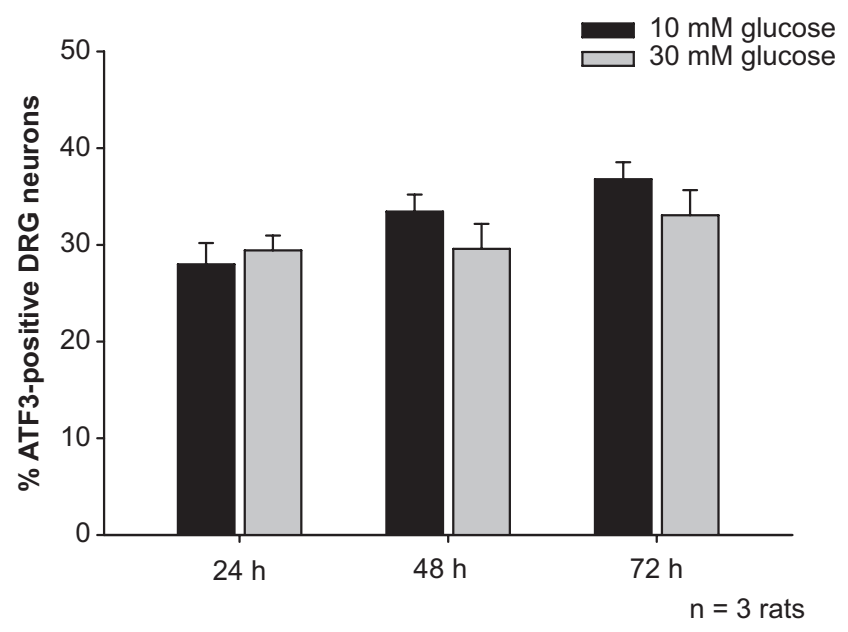

Figure 2 Determination of optimal glucose concentration in control conditions. Dorsal root ganglion neurons were cultured in medium containing 10 or $30 \mathrm{mM}$ glucose for 24,48 , and 72 hours. No significant differences were observed in ATF3 expression. Note: Shown is the mean of six wells from three rats \pm standard error of the mean. Abbreviations: ATF3, activating transcription factor 3; DRG, dorsal root ganglion.

quantified after a one-day culture period. The results show a time-dependent increase in neuronal stress response after streptozocin injection (Figure 4). One week after streptozocin treatment, a slight but not statistically significant increase in ATF3 expression was observed compared with DRG neurons isolated from age-matched control rats. The ATF3 level was significantly increased four weeks after streptozocin injection and rose further until week 16. At this point, there was a three-fold increase in ATF3-positive neurons.

To confirm that the increased ATF3 expression seen in DRG neurons isolated from diabetic rats was not due to a

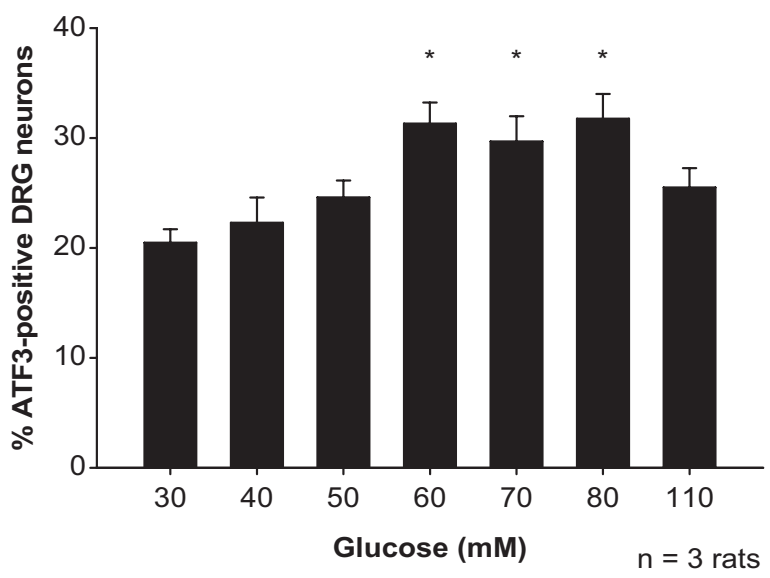

Figure 3 Neuronal stress in DRG neurons in hyperglycemic culture conditions. DRG neurons were treated for 24 hours with different concentrations of glucose. An increase in ATF3 expression was seen with doses between 60 and $80 \mathrm{mM}$ glucose.

Note: Shown is the mean of six wells from three rats \pm standard error of the mean $(* P<0.05)$.

Abbreviations: ATF3, activating transcription factor 3; DRG, dorsal root ganglion. 


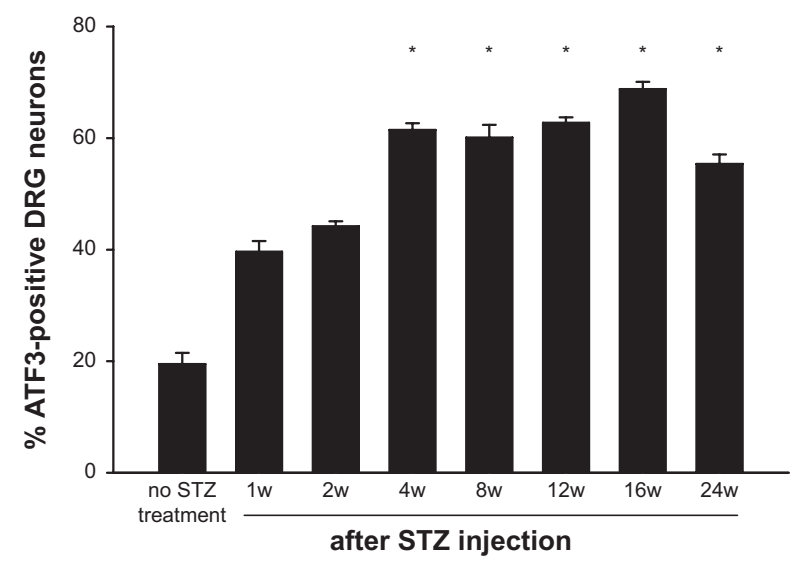

$\mathrm{n}=3$ rats/timepoint

Figure 4 Neuronal stress at different time points after streptozocin injection. Dorsal root ganglion neurons were isolated before streptozocin injection and at weeks I, 2, 4, 8, 12, 16, and 24 after streptozocin treatment. ATF3 expression is increased in dorsal root ganglion cultures isolated from 4-24-week diabetic rats. Note: Shown is the mean percentage of six wells from three rats \pm standard error of the mean $(* P<0.05)$.

Abbreviations: ATF3, activating transcription factor 3; DRG, dorsal root ganglion; STZ, streptozocin.

higher vulnerability of diabetic neurons to the isolation procedure, we did an RT-PCR analysis of whole DRG neurons from four-week diabetic rats (ie, four weeks after streptozocin injection) and compared the results with those from agematched control animals. DRG neurons from levels L4, L5, and L6 were dissected from the spinal column and immediately frozen. RNA extraction, cDNA preparation, and RT-PCR analysis were carried out for ATF3, as well as for other stressrelated genes, ie, c-jun and c-fos. The results show an increase in ATF3, c-jun, and c-fos in whole DRG neurons from diabetic rats when compared with control animals, which was significant for ATF3 and c-jun (Figure 5). This indicates that there was indeed higher neuronal stress in DRG neurons from diabetic rats, and that this increase was not due to a higher vulnerability to the isolation procedure. The level of c-jun in control animals (14.44 \pm 0.84$)$ was four times higher when compared with ATF3 expression in control rats $(3.40 \pm 0.31)$, making ATF3 a more specific marker for stress.

\section{Pharmacological modulation of acute hyperglycemia-induced ATF3 expression in vitro}

DRG neurons isolated from nondiabetic rats were pretreated with different concentrations of GDNF $(0.1-10.0 \mathrm{ng} / \mathrm{mL})$, gabapentin $(10-250 \mu \mathrm{M})$, and carbamazepine $(10-250 \mu \mathrm{M})$. After four hours, hyperglycemia was induced by incubating the cells in $60 \mathrm{mM}$ glucose for 24 hours. The percentage of

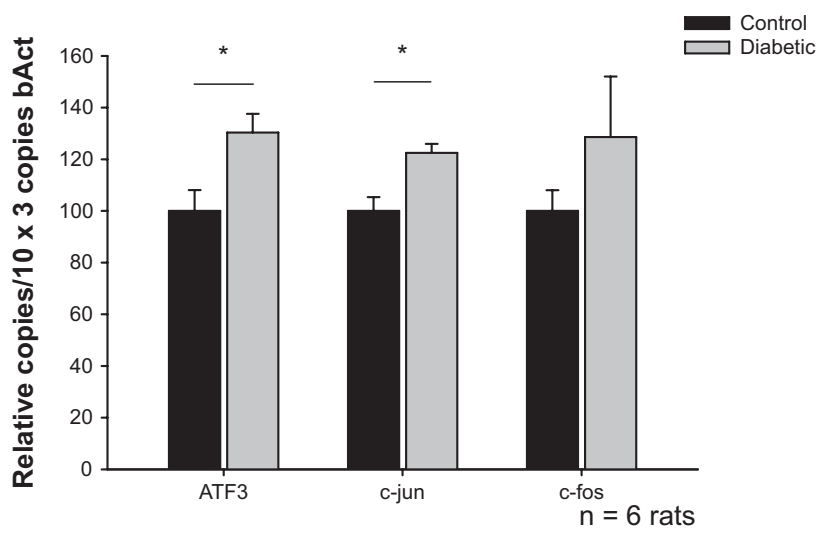

Figure 5 Real-time polymerase chain reaction analysis of dorsal root ganglion cultures from L4, L5, and L6 for the genes ATF3, c-jun, and c-fos. Dorsal root ganglion neurons were dissected from the spinal column of four-week diabetic rats and used for real-time polymerase chain reaction analysis. There is an increase in the ATF3, c-jun, and c-fos genes in whole dorsal root ganglion neurons isolated from diabetic rats, which is significant for ATF3 and c-jun.

Note: Shown is the mean number of copies/ $10 \times 3$ copies relative to control values bAct of three rats $(* P<0.05)$.

Abbreviation: ATF3, activating transcription factor 3 .

ATF3-positive neurons increased from $27.2 \pm 2.57$ in controls to $59.6 \pm 4.92$ in hyperglycemic cultures, ie, a $126 \%$ relative increase in ATF3-positive DRG neurons was observed. GDNF significantly blocked the increased ATF3 levels at doses of $1.0-0.1 \mathrm{ng} / \mathrm{mL}$ (Figure 6), indicating that GDNF is able to limit glucose-induced neuronal stress levels. DRG neurons exposed to hyperglycemia and pretreated with gabapentin or carbamazepine for four hours did not show a significant reduction in the percentage of ATF3-positive DRG neurons.

\section{Pharmacological modulation of diabetes- induced ATF3 expression ex vivo}

Next we evaluated the effect of GDNF, gabapentin, and carbamazepine ex vivo on the stress response of DRG neurons in eight-week diabetic rats and controls. DRG neurons were isolated and treated immediately with different concentrations of the respective substances for 24 hours. The percentage of ATF3-positive DRG neurons increased from $29.9 \pm 2.5$ in controls to $54.7 \pm 0.9$ in diabetic rats, ie, a $75 \%$ relative increase in ATF3 was observed. Treating DRG neurons with gabapentin or carbamazepine $(100-250 \mu \mathrm{M})$ did not reduce the percentage of ATF3-positive DRG neurons, whereas GDNF significantly reduced diabetes-induced ATF3 expression at doses of 10 and $1 \mathrm{ng} / \mathrm{mL}$ (Figure 7). This indicates that in vitro treatment with GDNF can effectively diminish diabetes-associated neuronal stress, whereas gabapentin and carbamazepine are not efficacious in reducing ATF3 levels of streptozocin-isolated DRG neurons treated ex vivo. 


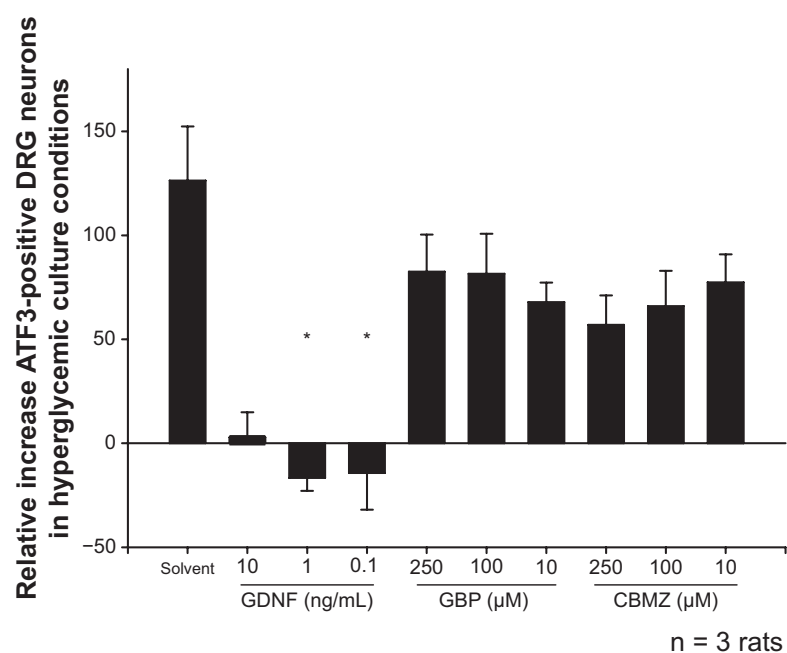

Figure 6 Effect of in vitro pretreatment with GDNF, GBP, and CBMZ on glucose-induced neuronal stress. DRG neurons were treated for four hours with different concentrations of GDNF, GBP, and CBMZ prior to addition of $60 \mathrm{mM}$ glucose. The ATF3 expression is decreased in GDNF-treated cultures, whereas GBP and CBMZ treatment has no significant effect on ATF3 levels.

Note: Shown is the mean percentage relative to control values obtained from six wells from three rats \pm standard error of the mean $(* P<0.05)$.

Abbreviations: ATF3, activating transcription factor 3; CBMZ, carbamazepine; DRG, dorsal root ganglion; GBP, gabapentin; GDNF, glial cell line-derived neurotrophic factor.

\section{Effect of in vivo GDNF treatment on the diabetes-induced ATF3 expression}

To confirm further the protective effect of GDNF on diabetesinduced neuronal stress, diabetic rats were treated with GDNF in vivo before DRG isolation and ATF3 sampling. Ten-week-old diabetic rats received subplantar injections of either GDNF $1 \mathrm{mg}$ $\mathrm{mL}$ or phosphate-buffered saline into the left hind paw for three consecutive days. DRGs from L4, L5, and L6 on the left side were then dissected from the spinal column, cultured for 24 hours, and used for ATF3 quantification. Diabetic rats treated with phosphate-buffered saline showed significantly higher ATF3 levels in DRG cultures when compared with nondiabetic control rats, whereas GDNF treatment significantly reduced ATF3 expression in DRG cultures from diabetic animals (Figure 8).

\section{Effect of GDNF on pain hypersensitivity in diabetic rats in vivo}

Because GDNF treatment was able to block hyperglycemiainduced and diabetes-induced ATF3 expression in DRG neurons, we investigated whether this protective effect also occurs in diabetes-induced pain hypersensitivity in vivo. Diabetic rats received subplantar injections of GDNF $1 \mathrm{mg} / \mathrm{mL}$ or phosphate-buffered saline in the left hind paw on days 17, 18, and 19 after streptozocin injection. The paw pressure test was used to investigate mechanical

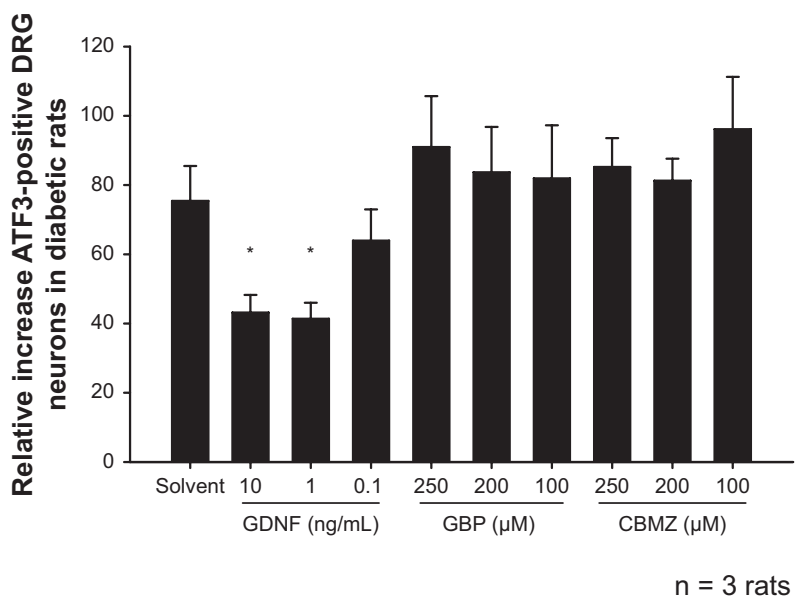

Figure 7 Effect of in vitro GDNF, GBP, and CBMZ treatment on diabetes-induced neuronal stress. DRG neurons were isolated from control and diabetic rats and treated with different concentrations of GDNF, GBP, and CBMZ. Treating DRG cultures from diabetic rats with GDNF decreased ATF3 expression, whereas GBP and CBMZ treatment had no stress-modulating effects.

Note: Shown is the mean percentage relative to control values of eight wells from three rats \pm standard error of the mean $(* P<0.05)$.

Abbreviations: ATF3, activating transcription factor 3; CBMZ, carbamazepine; DRG, dorsal root ganglion; GBP, gabapentin; GDNF, glial cell line-derived neurotrophic factor.

hyperalgesia, starting one day before diabetes induction and continuing until four weeks after streptozocin injection. A decrease in the withdrawal threshold was observed in rats two weeks after streptozocin injection when compared with age-matched control animals, indicating pain hypersensitivity in diabetic rats (Figure 9). When the diabetic animals were treated with GDNF, a significant increase in withdrawal pressure was observed when compared with the diabetic controls, being significant from days 2-6 after the first GDNF injection. These results indicate a protective effect of GDNF on diabetes- induced pain hypersensitivity, thereby further confirming our in vitro results.

\section{Discussion}

The present study shows a significant increase in neuronal stress in DRG neurons cultured in hyperglycemic conditions, starting from a concentration of $60 \mathrm{mM}$ glucose. The effect of high glucose concentrations on ATF3 expression in adult DRG cultures has not been investigated previously. However, earlier studies have investigated cell death in embryonic sensory neurons exposed to high glucose concentrations. Hyperglycemia (45 $\mathrm{mM}$ glucose) has been shown to trigger oxidative stress in DRG cultures by increasing the production of reactive oxygen species, resulting in apoptosis. ${ }^{9,33,34}$ Russell et $\mathrm{al}^{35}$ further indicated that mitochondria are associated with glucose-induced programmed cell death in 


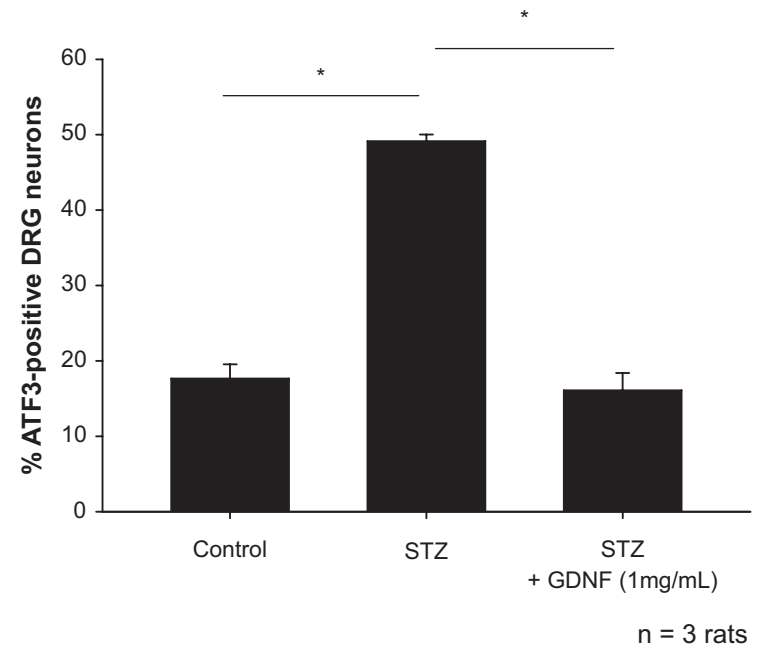

Figure 8 Effect of in vivo GDNF treatment on diabetes-induced stress. Ten-week diabetic rats were treated with GDNF or PBS in the left hind paw for three successive days. Afterwards, DRGs were isolated from L4, L5, and L6, and cultured. Control diabetic rats showed significantly higher ATF3 expression when compared with control animals, whereas the ATF3 level was normalized again in diabetic animals treated with GDNF.

Note: Shown is the mean percentage of six wells from three rats \pm standard error of the mean $(* P<0.05)$.

Abbreviations: ATF3, activating transcription factor 3; CBMZ, carbamazepine; DRG, dorsal root ganglion; GBP, gabapentin; GDNF, glial cell line-derived neurotrophic factor.

neurons, and showed that high glucose induces mitochondrial swelling and depolarization in sensory neurons. This not only leads to generation of reactive oxygen species, but also to partial depletion of ATP, an event that is coupled with apoptosis.

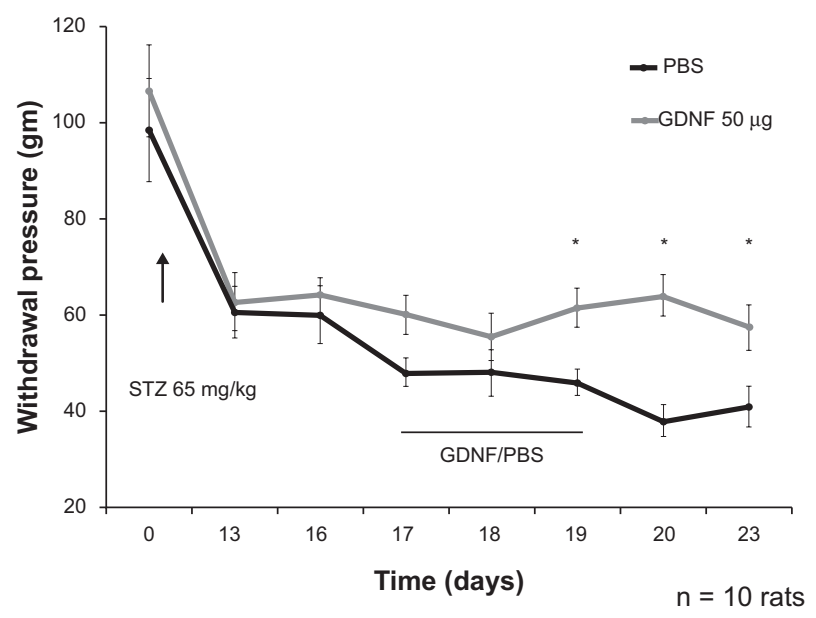

Figure 9 Effect of GDNF treatment on diabetes-induced pain hypersensitivity in vivo. Two weeks diabetic rats were treated with GDNF or PBS in the left hind paw for three successive days. The paw pressure test was used to investigate mechanical hyperalgesia. GDNF was able to improve the diabetes-induced pain hypersensitivity when compared with the control diabetic animals.

Note: Shown is the mean value from 10 rats $(* P<0.05)$.

Abbreviations: STZ, streptozocin; GDNF, glial cell line-derived neurotrophic factor; PBS, phosphate-buffered saline.
The effects of hyperglycemia are more difficult to investigate in adult sensory neurons. Gumy et $\mathrm{al}^{30}$ and Zherebitskaya et $\mathrm{al}^{31}$ were unable to show differences in neuronal death and oxidative stress over a four-week period in sensory neuron cultures from adult rats exposed to hyperglycemia (50-60 mM glucose). Differences in culture conditions between embryonic and adult cell cultures may play a role and, in particular, the presence of non-neuronal cells in the adult cultures may be protective. However, it is useful to investigate adult sensory neurons as an in vitro model because this is closer to the in vivo situation in animals and humans. Furthermore, cultured embryonic sensory neurons are phenotypically different from adult sensory neurons, and are dependent on neurotrophic factor-derived support for survival, ${ }^{36}$ which makes it difficult to study the effect of growth factors in this culture model. Quantification of ATF3 expression in adult sensory neurons appears to be a valuable tool to evaluate cellular dysfunction. ATF3 is an immediate early gene which is expressed immediately after cell injury. Upregulation of ATF3 after axotomy has been shown to stimulate axonal outgrowth and cell survival in an attempt to rescue the injured neuron. ${ }^{37-39}$ Because ATF3 is expressed before other damage to the cell occurs, it is a good early marker to quantify cellular stress.

In diabetic rats, a higher ATF3 expression was observed in DRG neurons starting one week after a single streptozotocin injection of $65 \mathrm{mg} / \mathrm{kg}$ intraperitoneally and reaching significance four weeks after streptozocin injection. These results correlate with in vivo testing in which streptozocin rapidly produced a marked effect. Forty-eight hours after streptozocin injection, rats were already hyperglycemic and showed physical signs of diabetes. Tactile allodynia is evident six days after streptozocin injection, and mechanical hyperalgesia develops after $9-13$ days. ${ }^{40}$ Histological investigation of DRGs isolated from diabetic rats have also revealed several pathological changes four weeks after streptozocin injection. Russell et $\mathrm{al}^{9}$ showed condensation of chromatin, shrinkage of the nucleus and cell cytoplasm, and ballooned mitochondria, with disruption of the inner cristae structure in DRG neurons, all of which are early signs of apoptosis. Srinivasan et $\mathrm{al}^{41}$ further confirmed these findings by demonstrating apoptosis and mitochondrial depolarization in DRG neurons isolated from diabetic rats 4-6 weeks after streptozocin injection. The high ATF3 expression and apoptotic changes in DRG neurons, together with the altered sensory function in diabetic animals, may indicate the onset of diabetic peripheral neuropathy. 
Our findings complement those of Wright et al, ${ }^{42}$ who found a significantly higher level of ATF3 expression in DRG neurons of diabetic mice when compared with controls. ATF3 expression was apparent three weeks after streptozocin injection, while one-week diabetic mice showed no ATF3 expression. This correlates with our observations, which showed a significantly increased ATF3 expression in rat DRG cultures starting from four weeks after streptozocin injection. Due to the different experimental methods used in our study, there was little difference in ATF3 expression seen in the DRG neurons from nondiabetic rats. We observed 19.48\% ATF3-positive neurons in our control rats while Wright et al ${ }^{42}$ observed few, if any, ATF3-positive DRG neurons in their control mice. Wright et al dissected only L4 and L5 DRGs, which were immediately fixed and used for histological research. In our study, we used DRG neurons which had been cultured for at least 24 hours before fixation and immunocytochemistry. Isolation and culture of DRG neurons is in itself a stressful event for these cells, explaining the higher ATF3 levels in control cultures. ${ }^{43}$ In addition to this, a higher vulnerability of the DRG neurons from diabetic rats to the isolation and culture conditions might also contribute to the observed increase in ATF3 expression.

In contrast with Wright et al, ${ }^{42}$ a study by Dahlin et al ${ }^{44}$ concluded that diabetes does not in itself cause an increase in ATF3 expression, but it does enhance the expression of ATF3 in response to nerve injury. To investigate whether the higher ATF3 expression seen in DRG cultures from diabetic rats is not just due to a higher vulnerability of the neurons to the isolation procedure, we undertook an RT-PCR analysis of whole-mounted DRGs for the stress-related genes, ATF3, c-jun, and c-fos. Four weeks after streptozocin injection, there was a significantly higher level of ATF3 and c-jun seen in whole-mounted L4, L5, and L6 DRG neurons isolated from diabetic rats when compared with age-matched controls. This confirms the results seen in DRG cultures, indicating that diabetes does increase the stress level in DRG neurons.

Previous work has shown that growth factors promote survival of peripheral neurons and induce neuronal regeneration in in vitro and in vivo models of neurological injury and disease. GDNF has attracted considerable attention because of its ability to support the survival of dopamine-containing neurons which degenerate in Parkinson's disease, and is therefore an attractive agent for the treatment of this disease. ${ }^{12}$ GDNF is also a potent survival factor for spinal motor neurons, and so could have clinical importance for the treatment of amyotrophic lateral sclerosis. ${ }^{13}$ GDNF has been found to hold promise as a potential agent in the treatment of diabetic peripheral neuropathy. Akkina et $\mathrm{a}^{45}$ demonstrated that GDNF administration to diabetic mice reversed diabetes-induced deficits in the spinal cord. Furthermore, Christianson et $\mathrm{a}^{46}$ reported that GDNF might be effective in improving the cutaneous innervation deficits that occur in sensory neuropathies. Because of these known neuroprotective properties, the effect of GDNF on diabetes-induced neuronal stress in rat DRG cultures was evaluated in the present study. We showed that pretreatment with GDNF inhibited the increase in ATF3 expression after exposure of DRG neurons to hyperglycemia in vitro. Furthermore, GDNF treatment of DRG cultures from diabetic rats ex vivo and subplantar injections of GDNF in vivo decreased diabetesinduced ATF3 levels in DRG neurons.

This study is the first to show the beneficial effect of GDNF on the neuronal stress response in conditions of in vitro diabetic peripheral neuropathy. Previous studies illustrated the effect of GDNF on ATF3 expression after nerve injury, but no studies were conducted in models of diabetes. Averill et a $1^{47}$ showed that GDNF partially prevents the upregulation of ATF3 in DRG neurons after unilateral sciatic nerve axotomy in rats. A similar study was conducted by Wang et al, ${ }^{48}$ in which they induced neuronal injury by spinal nerve ligation in rats and evaluated the effect of GDNF on the injury-induced neuronal damage. Like in the present study, the same neuroprotective effects for GDNF were found.

Gabapentin and carbamazepine are anticonvulsant drugs currently in use for the treatment of neuropathic pain and peripheral neuropathies, including diabetic neuropathy. In contrast with GDNF, these drugs act only symptomatically. Few studies have investigated the effects of gabapentin and carbamazepine in DRG cultures, so we compared the effect of both these anticonvulsants on neuronal stress with that of GDNF. Our experiments show that both gabapentin and carbamazepine have limited neuroprotective ability, which is in line with their presumed mechanism of action, whereas GDNF is able to reduce diabetes-induced neuronal stress.

In summary, our results show increased ATF3 expression in DRG neurons in hyperglycemic culture conditions and in DRGs isolated from diabetic rats. Inhibition of neuronal stress could be important in the development of diseasemodifying therapy for diabetic peripheral neuropathy. GDNF, a substance with known neuroprotective properties, was able to reduce diabetes-induced neuronal stress, while 
gabapentin and carbamazepine, currently in use for the treatment of neuropathic pain, showed only limited effects.

\section{Conclusion}

Overall, we can conclude that growth factors show promise as neurotrophic agents in the treatment of diabetic peripheral neuropathy, whereas gabapentin and carbamazepine have no direct protective effect on sensory neurons. These results warrant further research to confirm the disease-modifying potential of GDNF in peripheral neuropathy. Furthermore, this study indicates that immunocytochemistry for ATF3 expression is a reliable marker of stress in DRG cultures, and allowing pharmacological evaluation of compounds in this model.

\section{Disclosure}

The authors report no conflicts of interest in this work.

\section{References}

1. Pirart J. Diabetes mellitus and its degenerative complications: A prospective study of 4,400 patients observed between 1947 and 1973. Diabetes Care. 1978;1:168-188.

2. Thomas PK, Tomlinson DR. Diabetic and hypoglycaemic neuropathy. In: Dyck PJ, Thomas PK, Griffin JW, Low PA, Poduslo JF, editors. Peripheral Neuropathy. Philadelphia, PA: WB Saunders Co; 1992.

3. Yagihashi S. Pathology and pathogenetic mechanisms of diabetic neuropathy. Diabetes Metab Rev. 1996;11:193-225.

4. The Diabetes Control and Complications Trial Research group. The effect of intensive treatment of diabetes on the development and progression of long-term complications in insulin-dependent diabetes mellitus. N Engl J Med. 1993;329:977-986.

5. The Diabetes Control and Complications Trial Research Group. The effect of intensive diabetes therapy on the development and progression of neuropathy. Ann Intern Med. 1995;122:561-568.

6. Sima AAF. New insights into the metabolic and molecular basis for diabetic neuropathy. Cell Mol Life Sci. 2003;60:2445-2464.

7. Leinninger GM, Vincent AM, Feldman EL. The role of growth factors in diabetic peripheral neuropathy. J Peripher Nerv Syst. 2004;9: 26-53.

8. Russell JW, Windebank AJ. Electrophysiological and pathological characteristics of suramin induced neuropathy. Neurology. 1993; 43:A174.

9. Russell JW, Sullivan KA, Windebank AJ, Herrmann DN, Feldman EL. Neurons undergo apoptosis in animal and cell culture models of diabetes. Neurobiol Dis. 1999;6:347-363.

10. Backonja M. Use of anticonvulsants for the treatment of neuropathic pain. Neurology. 2002;59:S14-S17.

11. Jensen TS. Anticonvulsants in neuropathic pain: Rationale and clinical evidence. Eur J Pain. 2002;6:61-68.

12. Lin LH, Doherty DH, Lile JD, Bektesh S, Collins F. GDNF: A glial cell line-derived neurotrophic factor for midbrain dopaminergic neurons. Science. 1993;260:1130-1132.

13. Henderson CE, Phillips HS, Pollock RA, et al. GDNF: A potent survival factor for motoneurons present in peripheral nerve and muscle. Science. 1994;266:1062-1064.

14. Molliver DC, Wright DE, Leitner ML, et al. IB4-binding DRG neurons switch from NGF to GDNF dependence in early postnatal life. Neuron. 1997; 19:849-861.

15. Dickernson AH, Matthews EA, Suzuki R. Neurobiology of neuropathic pain: Mode of action of anticonvulsants. Eur J Pain. 2002;6:51-60.
16. Curran MP, Wagstaff AJ. Gabapentin in postherpetic neuralgia. CNS Drugs. 2003;17:975-982.

17. Chen BP, Wolfgang CD, Hai T. Analysis of ATF3, a transcription factor induced by physiological stresses and modulated by gadd153/chop 10 . Mol Cell Biol. 1996;16:1157-1168.

18. Yin T, Sandhu G, Wolfgang CD, et al. Tissue-specific pattern of stress kinase activation in ischemic/reperfused heart and kidney. $\mathrm{J}$ Biol Chem. 1997;272:19943-19950.

19. Hai T, Wolfgang CD, Marsee DK, Allen AE, Sivaprasad U. ATF3 and stress responses. Gene Expression. 1999;7:321-335.

20. Tsujino H, Kondo E, Fukuoka T, et al. Activating transcription factor 3 (ATF3) induction by axotomy in sensory and motoneurons: A novel neuronal marker of nerve injury. Mol Cell Neurosci. 2000;15: $170-182$.

21. Hai T, Curran T. Cross-family dimerization of transcription factors Fos/Jun and ATF/CREB alters DNA binding specificity. Proc Natl Acad Sci U S A. 1991;88:3720-3724.

22. Obata K, Yamanaka H, Fukuoka T, et al. Contribution of injured and uninjured dorsal root ganglion neurons to pain behavior and the changes in gene expression following chronic constriction injury of the sciatic nerve in rats. Pain. 2003;101:65-77.

23. Huang WL, Robson M, Liu C, et al. Spinal cord compression and dorsal root injury cause up-regulation of activating transcription factor-3 in large-diameter dorsal root ganglion neurons. Eur J Neurosci. 2006;23: 273-278.

24. Shortland PJ, Baytug B, Krzyzanowska A, McMahon SB, Priestley JV, Averill S. ATF3 expression in L4 dorsal root ganglion neurons after L5 spinal nerve transaction. Eur J Neurosci. 2006;23:265-373.

25. Saito H, Dahlin LB. Expression of ATF3 and axonal outgrowth are impaired after delayed nerve repair. BMC Neurosci. 2008;9:88.

26. Bráz JM, Basbaum AI. Differential ATF3 expression in dorsal root ganglion neurons reveal the profile of primary afferents engaged by diverse noxious chemical stimuli. Pain. 2010;150:290-301

27. Baudet C, Mikaels A, Westphal H, Johansen J, Johansen TE, Ernfors P. Positive and negative interactions of GDNF, NTN and ART in developing sensory neuron subpopulations, and their collaboration with neurotrophins. Development. 2000;127:4335-4344.

28. Madduri S, Papaloïzos M, Gander B. Synergistic effect of GDNF and NGF on axonal branching and elongation in vitro. Neurosci Res. 2009; 65:88-97.

29. Perucca E. Is there a role for therapeutic drug monitoring of new anticonvulsants? Clin Pharmacokinet. 2000;38:191-204.

30. Gumy LF, Bampton ETW, Tolkovsky AM. Hyperglycaemia inhibits Schwann cell proliferation and migration and restricts regeneration of axons and Schwann cells from adult murine DRG. Mol Cell Neurosci. 2008:37:298-311.

31. Zherebitskaya E, Akude E, Smith DR, Fernyhough P. Development of selective axonopathy in adult sensory neurons isolated from diabetic rats: Role of glucose-induced oxidative stress. Diabetes. 2009;58: 1356-1364.

32. Vincent AM, Olzmann JA, Brownlee M, Sivitz WI, Russell JW. Uncoupling proteins prevent glucose-induced neuronal oxidative stress and programmed cell death. Diabetes. 2004;53:726-734.

33. Vincent AM, Russell JW, Low P, Feldman EL. Oxidative stress in the pathogenesis of diabetic neuropathy. Endocr Rev. 2004;25:612-628.

34. Vincent AM, McLean LL, Backus C, Feldman EL. Short-term hyperglycemia produces oxidative damage and apoptosis in neurons. FASEB J. 2005;19:638-640.

35. Russell JW, Golovoy D, Vincent AM, et al. High glucose-induced oxidative stress and mitochondrial dysfunction in neurons. FASEB $J$. 2002;16:1738-1748.

36. Lindsay RM. Nerve growth factors (NGF, BDNF) enhance axonal regeneration but are not required for survival of adult sensory neurons. J Neurosci. 1988;8:2394-2405.

37. Pearson AG, Gray CW, Pearson JF, Greenwood JM, During MJ, Dragunow M. ATF3 enhances c-Jun-mediated neurite sprouting. Mol Brain Res. 2003;120:38-45. 
38. Lindwall C, Kanje M. The Janus role of c-jun: Cell death versus survival and regeneration of neonatal sympathetic and sensory neurons. Exp Neurol. 2005;196:184-194.

39. Seijffers R, Allchorne AJ, Woolf CJ. The transcription factor ATF-3 promotes neurite outgrowth. Mol Cell Neurosci. 2006;32:143-154.

40. Hoybergs YMJJ, Meert TF. The effect of low-dose insulin on mechanical sensitivity and allodynia in type I diabetes neuropathy. Neurosci Lett. 2007;417:149-154.

41. Srinivasan S, Stevens M, Wiley JW. Diabetic peripheral neuropathy. Evidence for apoptosis and associated mitochondrial dysfunction Diabetes. 2000;49:1932-1938.

42. Wright DE, Ryals JM, McCarson KE, Christianson JA. Diabetes-induced expression of activating transcription factor 3 in mouse primary sensory neurons. J Peripher Nerv Syst. 2004;9:242-254.

43. Aoki Y, An HS, Takahashi K, et al. Axonal growth potential of lumbar dorsal root ganglion neurons in an organ culture system. Spine. 2007;32: 857-863.

44. Dahlin LB, Stenberg L, Luthman H, Thomsen NOB. Nerve compression induces activating transcription factor 3 in neurons and Schwann cells in diabetic rats. Neuroreport. 2008;19:987-990.
45. Akkina SK, Patterson CL, Wright DE. GDNF rescues nonpeptidergic unmyelinated primary afferents in streptozotocin-treated diabetic mice. Exp Neurol. 2001;167:173-182.

46. Christianson JA, Riekhof JT, Wright DE. Restorative effects of neurotrophin treatment on diabetes-induced cutaneous axon loss in mice. Exp Neurol. 2003;179:188-199.

47. Averill S, Michael GJ, Shortland PJ, et al. NGF and GDNF ameliorate the increase in ATF3 expression which occurs in dorsal root ganglion cells in response to peripheral nerve injury. Eur J Neurosci. 2004;19: 1437-1445.

48. Wang R, Guo W, Ossipov MH, Vanderah TW, Porreca F, Lai J. Glial cell line-derived neurotrophic factor normalizes neurochemical changes in injured dorsal root ganglion neurons and prevents the expression of experimental neuropathic pain. Neuroscience. 2003;121:815-824.
Journal of Pain Research

\section{Publish your work in this journal}

The Journal of Pain Research is an international, peer-reviewed, open access, online journal that welcomes laboratory and clinical findings in the fields of pain research and the prevention and management of pain. Original research, reviews, symposium reports, hypothesis formation and commentaries are all considered for publication.

\section{Dovepress}

The manuscript management system is completely online and includes a very quick and fair peer-review system, which is all easy to use. Visit http://www.dovepress.com/testimonials.php to read real quotes from published authors. 